\title{
DEVELOPMENT OF A COMPUTATIONAL MULTI-PHYSICAL FRAMEWORK FOR THE USE OF NONLINEAR EXPLICIT APPROACH IN THE ASSESSMENT OF CONCRETE STRUCTURES AFFECTED BY ALKALI-AGGREGATE REACTION
}

\author{
BEN FTIMA M. ${ }^{*}$, SADOUKI H. ${ }^{\dagger}$ AND BRÜHWILER E. ${ }^{\dagger \dagger}$ \\ "École Polytechnique de Montréal \\ B.O. Box 6079, Station Centre-ville \\ e-mail: mahdi.ben-ftima@polymtl.ca \\ École Polytechnique Fédérale de Lausanne \\ EPFL ENAC IIC MCS, GC B2 386, Station 18 \\ e-mail: hamid.sadouki@epfl.ch \\ ${ }^{\dagger}$ École Polytechnique Fédérale de Lausanne \\ EPFL ENAC IIC MCS, GC B2 386, Station 18 \\ e-mail: eugen.bruehwiler@epfl.ch
}

Key words: Alkali-aggregate reaction, multi-physical, concrete model, nonlinear, explicit.

\begin{abstract}
This paper proposes an innovative methodology for the use of the explicit approach in the assessment of concrete structures affected by alkali-aggregate reaction (AAR). Efficiency of the explicit approach has been proven in previous works for the case of large concrete structural models with high degree of nonlinearity. In the proposed methodology, the strain is decomposed into mechanical, thermal, creep, shrinkage and AAR strain components. The AAR component is computed according to Saouma and Perotti model [1] for the anisotropic distribution of the volumetric expansion and according to Larive model [2] for the AAR kinetics. One advantage of the approach is that it can be used with any existing concrete model that has undergone a rigorous verification and validation $(\mathrm{V} \& \mathrm{~V})$ process for the mechanical part. In this work the EPM3D concrete model [2] implemented as a user-subroutine in Abaqus-Explicit is used. The general methodology is based on three different finite element analyses: thermal implicit, hygral implicit and the final nonlinear multiphysical explicit analysis. An innovative formulation to address the problem of time scale difference between the implicit and explicit approaches is presented. A new incremental numerical formulation is presented to correctly handle the dependency of the AAR kinetics on the temperature field in case of cyclic temperature variation. A verification example is presented at the material level, along with qualitative assessment of the cracking pattern of an existing hydraulic structure affected by AAR. This last application at structural level demonstrates the efficiency of the suggested methodology and the feasibility within an industrial context.
\end{abstract}

\section{INTRODUCTION}

Alkali-aggregate reaction (AAR) is an acidbase chemical reaction known to occur in concrete for certain types of aggregates and under certain moisture, confinement and temperature conditions. Structural manifestation of this reaction is due to the expansive nature of the alkali-silica gel, product 
of this reaction, when it comes in contact with moisture. This internal expansion generally leads to micro-cracking, loss of strength and stiffness at the material level. Major distortions can occur at the structural level leading to functional problems of the onboard installation. As an example, expansion in hydraulic structures like dams and powerhouses can interfere with the operation of the flow gates or turbines. Macro-cracking and failure may occur when the deformations are prevented or when concrete structural components are unable to accommodate these large deformations.

Numerical models can be carried out to predict structural displacements, stresses and damage resulting from AAR. Finite elements linear analyses have been successfully applied to predict displacement field in structures and were validated with field monitored data (e.g. [4]). The analyses based on fictitious thermal loading conditions found on a trial-and-error basis using the past monitored data were successfully applied in the engineering community. These analyses predict quite reasonably the displacement field of structures affected by AAR but cannot be used to predict the stress field or the damage pattern. More recently, a new family of models called coupled chemo-mechanical models started to emerge ([5], [1], [6], [7], [8]). In these models, kinetics of the chemical reaction is simulated within the nonlinear mechanical constitutive model. Experimental works were performed in order to characterize the parameters needed in these models. Among these works, the experiments of Larive [2] and Multon [9] carried out at the LCPC Laboratory in France are of great importance since they characterized for the first time both the kinetics and the anisotropy of the swelling process depending on stress and environmental conditions. AAR in concrete structures is still considered as a complex phenomenon mainly due to its multi-physical nature. The lack of experiments to validate available numerical models on the element and structural level is obvious. Based on past investigations, it can be concluded that the major influencing factors that need to be considered within a constitutive model are: kinetics of the reaction and of gel swelling process, effects of humidity and temperature fields, effects of the stress field and confining conditions on the anisotropy of swelling, effects of cracking, intrinsic degradation of material and creep effects. Considering all of these aspects within a nonlinear finite element framework and for a real structural model can result in computational challenges and makes questionable the feasibility of these sophisticated analyses within a real industrial context. Furthermore, it is known that AAR results over time in a generalized cracking pattern and highly nonlinear behavior when compared to conventional mechanical loading. Cracking in concrete is handled numerically by the softening behavior on the stress-strain curve of the constitutive law which generally results in numerical difficulties with the conventional implicit and iterative numerical resolution algorithms. Non convergence issues can occur for large concrete models with no or light reinforcement as it is the case for example for hydraulic structures [10]. According to authors past experience, mesh refinement in a concrete model can result in a numerical damage pattern very close to the real cracking pattern even when the smeared crack approach is used. Convergence difficulties may again arise when using finer mesh and force analyst to use a coarse mesh making difficult the comparison between model and reality. The objective of this work is to develop a new computational framework suitable for the multi-physical simulation of AAR within concrete structures. For the first time, the explicit quasi-static approach which was initially developed within metal forming field is used. The approach is based on three constitutive analyses: thermal analysis, hygral analysis and a final multiphysical analysis. An innovative time scaling technique is presented in order to deal with the 
time scale difference between these three analyses. The efficiency and feasibility of the approach are demonstrated via a real industrial example of a hydraulic structure. This work is a part of a larger long term research program that addresses the issue of practical use of nonlinear finite elements for the assessment of hydraulic structures affected by AAR.

\section{EXPLICIT QUASI-STATIC APPROACH}

The explicit dynamics approach has been developed and successfully applied in the industrial field of metal forming, at the beginning of the nineties [11]. Following this approach, the nonlinear problem is solved in a dynamic manner and conventional nodal forces are converted into inertia forces by assigning lumped masses into the nodes. With comparison to the conventional implicit approach, no iterations are performed, the algorithm advances explicitly in time using a very small time increment to ensure stability. This increment $\Delta t$ depends on the smallest element of the mesh and can be as low as $10^{-5}$ to $10^{-7}$ fraction of the total analysis time. This approach has been successfully extended to the case of civil engineering concrete structures [10] and applied to the industry of hydraulic structures. The original nonlinear static problem is solved in a quasi-static manner by applying all the loads slowly enough with respect to the first mode, to ensure that kinetic energy is negligible compared to the internal strain energy. For a typical civil engineering structure with a fundamental period $T_{1}$ ranging from $0.05 \mathrm{sec}$ to $2 \mathrm{sec}$, this involves a period of analysis $t_{\text {exp }}$ ranging from $1 \mathrm{sec}$ to $40 \mathrm{sec}$ considering a ratio of 20 for $t_{\text {exp }} / T_{1}$. Hence, the approach is more efficient for stiff structures considering the small stable increment time. It was possible for the first time to analyse complex nonlinear models (nonlinear constitutive model and contact condition with friction) with more than three millions degrees of freedom. The parallel computing technique was investigated and benchmark tests showed that computation time can be almost inversely proportional to the number of cores used in the computation. Unlike implicit approach, the global model is divided into $\mathrm{N}$ domains corresponding to the number of cores available. This is possible in the explicit approach since there is no need to consider the assembly of the global stiffness matrix as it is the case in the conventional implicit approach. Figure 1 shows an example of domain subdivision of the structural model considered in this work, each color represents a domain. In this case computations are done on a conventional quadcore computer. The model has more than 140 000 elements.

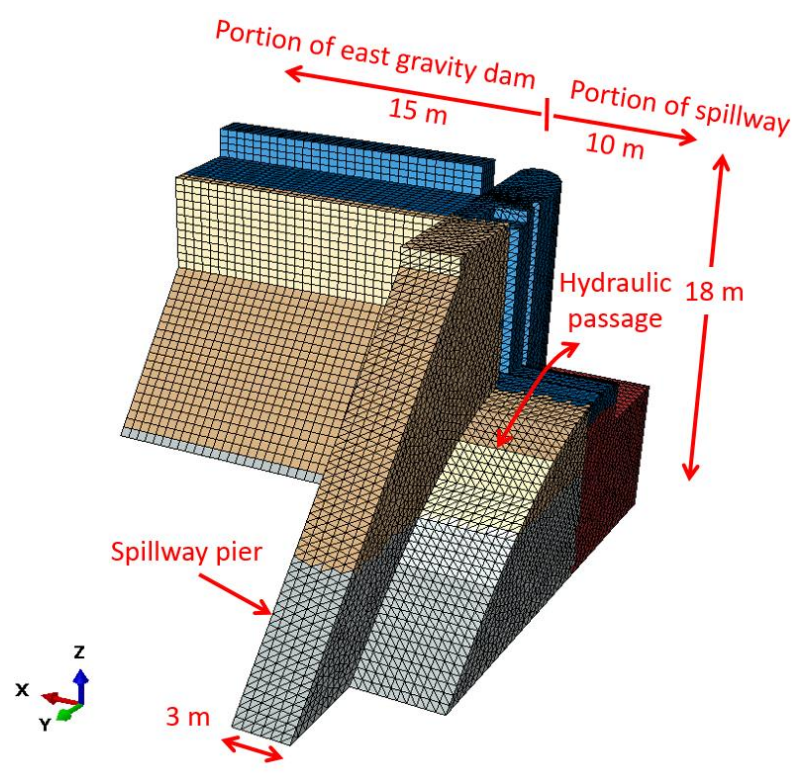

Figure 1: Finite element model of the hydraulic structure

\section{EPM3D CONCRETE MODEL}

The EPM3D concrete model [3] is a 3D constitutive model that uses the smeared-crack approach and can model tensile cracking and concrete crushing of concrete. EPM3D is an orthotropic 3D strain-rotating crack model. It was programmed under Abaqus-Explicit [12] using the VUMAT user-subroutine interface [13]. It can handle both unreinforced and reinforced concrete applications using, respectively, a $G_{f}$ law and tension-stiffening 
law for the post-cracking regime in the presence of reinforcement. As shown in Figure 2, the model was originally implemented as a purely mechanical constitutive law updating at each increment of time the stress tensor $\sigma_{1}$ and the state dependent variable vector $\mathrm{SDV}_{1}$, given the stress tensor $\sigma_{0}$ of the previous increment, the state dependent variable vector $\mathrm{SDV}_{0}$ of the previous increment and the given incremental strain tensor $\Delta \varepsilon$. Over the past ten years, the mechanical constitutive law of EPM3D has undergone a rigorous verification and validation process $(\mathrm{V} \& \mathrm{~V})$ and was used in the hydraulic structures and bridge engineering industries ([14] and [15]). More recently, the participation using this model was ranked the second out of sixty six within an international prediction contest organized by the University of Toronto on the deepest reinforced concrete beam ever tested (4 meters). Over the sixty six participants, only the two first entries predicted the failure loads of both the east and shear span and the west span within $10 \%$ of the experimental values [16]. A large coefficient of variation was observed during this contest though the simplicity of the geometry which clearly demonstrates that validation of a purely mechanical constitutive concrete is still an issue in the field of lightly reinforced or nonreinforced concrete structures. This finding supports the decision to include the AAR model within the existing mechanical constitutive law. To the best of author's knowledge, the only AAR model that can be implemented with an existing constitutive model and at the same time handles the major influencing factors cited in the introduction is the one proposed in [1]. This will be developed in the next section.

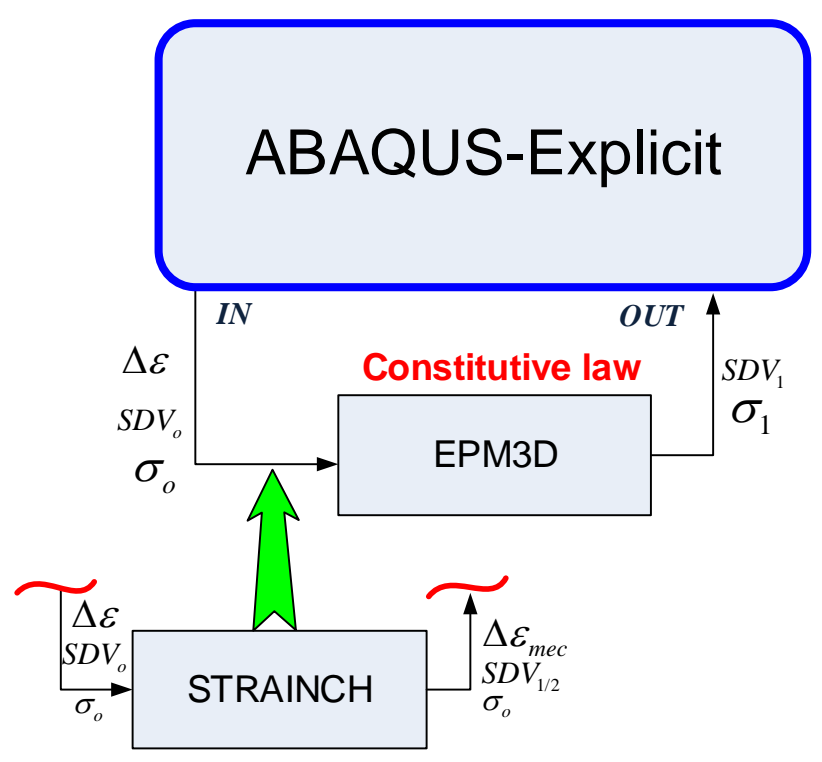

Figure 2: User subroutine implementation and modifications of the existing EPM3D constitutive law

\section{MULTI-PHYSICAL SIMULATION OF AAR IN CONCRETE STRUCTURES}

\subsection{Multi-physical approach}

The multi-physical approach developed in this work uses three different analyses as shown in Figure 3 and involves a sequential coupling between thermal, hygral and chemo/mechanical fields. Two transient thermal and hygral analyses are firstly performed in order to compute the thermal and relative humidity fields $\mathrm{T}(\mathrm{t})$ and $\mathrm{H}(\mathrm{t})$. The thermal analysis is a conventional heat transfer analysis involving conduction and convection heat transfer modes and body heat flux to simulate the exothermic hydration process. The hygral analysis is performed in a similar way using the analogy between heat and hygral flux and using the concept of diffusivity with unit values of densities and specific heat [17]. In both cases, the simulation period of time can be as large as the lifetime span $t_{\text {life }}$ of the structure. These two fields are than imported into the final multiphysical analysis which is actually the nonlinear structural analysis involving the modified EPM3D model. This last analysis involves computation over time of the stress field $\sigma(t)$ and the AAR kinetic progress 
parameter $\xi(t)$ using the notation and equations of Larive [2]. The first two analyses are performed in a conventional implicit framework. Even if the constitutive equation of the heat transfer law is nonlinear in the case of hygral analysis, due to the dependency of the diffusivity on the hygral field, no convergence difficulties are anticipated. In fact numerical difficulties arise only when a softening behavior exist in the constitutive law. The final analysis however is performed in the dynamic explicit framework. This difference between implicit and explicit frameworks involves a challenging problem of difference in time scales: in the order of several years for the implicit analyses and of few seconds in case of the explicit analyses. This problem will be treated in a later section.

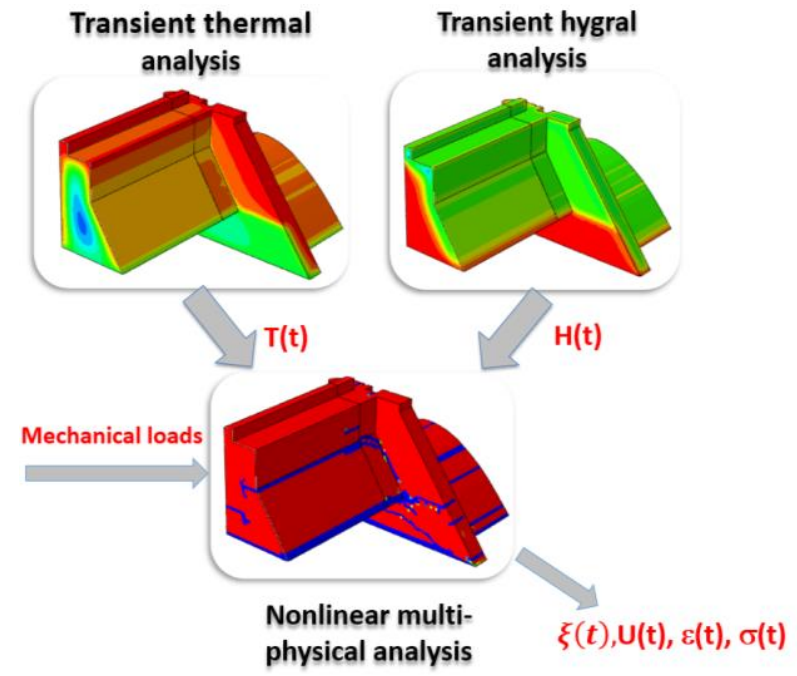

Figure 3: Multi-physical approach.

\subsection{Strain decomposition}

Similarly to the work of Pan et al. [18], the total strain is decomposed into mechanical, thermal, creep, shrinkage and AAR strains according to the following equation in the incremental form:

$$
\begin{aligned}
& \Delta \epsilon(t, T, H)=\Delta \epsilon_{\text {mec }}(t, T, H)+\Delta \epsilon_{t h}(t, T)+ \\
& \Delta \epsilon_{c r}\left(t, \sigma_{0}\right)+\Delta \epsilon_{s h}(t, H)+ \\
& \Delta \epsilon_{\text {aar }}\left(t, \xi, T, H, \sigma_{0}\right)
\end{aligned}
$$

This equation shows inter alia the coupling between the four fields considered in the study. As shown schematically in Figure 2, the methodology involves at each stable increment time, the computation of the mechanical strain increment from the total strain increment given by Abaqus-Explicit and the remaining thermal, creep, shrinkage and AAR strains as shown in Eq. 1. This is performed via the new subroutine STRAINCH shown in Figure 2. This subroutine additionally updates the state variables vector $S D V$ required for the mechanical constitutive law EPM3D.

The following equations are used for the estimation of thermal, creep, shrinkage and AAR strains:

\section{Thermal:}

$$
\Delta \epsilon_{t h}(t, T)=\alpha\left(T(t)-T_{i n i}\right) \delta
$$

where $\alpha$ is the coefficient of thermal expansion; $T_{\text {in } i}$ is the initial reference temperature and $\delta$ is the Konecker's delta.

\section{Creep:}

Kelvin-Voigt viscoelastic model (damper $\eta_{v e}$ and elastic spring $E_{v e}$ ) is used, similarly to Pan et al. 2013.

$$
\begin{aligned}
& \Delta \epsilon_{c r}\left(t, \sigma_{0}\right)=\left(e^{\frac{-E_{v e}}{\eta_{v e}}}-1\right)\left(\epsilon_{c r}(t-\Delta t)-\right. \\
& \left.\frac{[A] \sigma_{0}}{E_{v e}}\right)
\end{aligned}
$$

Where $[A]=$

$$
\left[\begin{array}{cccccc}
1 & -v & -v & & & \\
-v & 1 & -v & & 0 & \\
-v & -v & 1 & & & 0 \\
& & & 2(1+v) & 0 & 0 \\
& 0 & & 0 & 2(1+v) & 0 \\
& & & 0 & 0 & 2(1+v)
\end{array}\right]
$$

and $v$ is the Poisson's coefficient.

Shrinkage:

Similarly to [17]:

$$
\Delta \epsilon_{s h}(t, H)=\alpha_{s h}(H)(H(t)-H(t-\Delta t)) \delta
$$


where $\alpha_{s h}$ is the shrinkage coefficient that depends nonlinearly on the relative humidity field $\mathrm{H}$.

\section{AAR:}

Using the same notations as in [1]:

$$
\begin{aligned}
& \Delta \epsilon_{\text {aar }_{i}}\left(t, \xi, T, H, \sigma_{0}\right)= \\
& \Gamma_{t}\left(S D V_{0}\right) \cdot \Gamma_{c}\left(\sigma_{0}\right) \cdot g(H) \cdot W_{i}\left(\sigma_{0}\right) \cdot \Delta \xi\left(t, T, \sigma_{0}\right) \cdot \varepsilon_{v \infty}
\end{aligned}
$$

where $\mathrm{i}$ is a given stress principal direction, $\Gamma_{t}$ and $\Gamma_{c}$ are retardation factors that depend on the tensile, compressive damage and stress field of the material point in the previous increment, $g(H)=H^{8}$ is a reduction function that accounts for the influence of humidity, $W_{i}\left(\sigma_{0}\right)$ is the weighting for redistribution of volumetric AAR expansion in the principal direction i, depending on the stress field as described in [1], $\varepsilon_{v \infty}$ is the long term volumetric AAR strain and $\Delta \xi\left(t, T, \sigma_{0}\right)$ is the rate of the AAR reaction given by the following equation:

$\Delta \xi\left(t, T, \sigma_{0}\right)=\frac{e^{\frac{t}{\tau_{c}}}\left(e^{\left.\frac{\tau_{l}}{\tau_{c}}+1\right)}\right.}{\tau_{c}\left(e^{\left.\frac{\tau_{l}}{\tau_{c}}+1\right)}\right.} \Delta t$

where $\tau_{l}=\tau_{l}(T)$ is the latency time that depends on temperature field and $\tau_{c}=$ $\tau_{c}\left(T, \sigma_{0}\right)$ is the characteristic time that depends on temperature and stress field as described in [1]. Hence, an incremental formulation of the kinetics of AAR in terms of $\Delta \xi$ is used in lieu of a total or secant formulation in terms of $\xi$, as recommended by [19]. However, special care need to be taken for the case of rapid cyclic variation of temperature as it will be shown in section 5 .

\subsection{Time scaling}

Two different time scales are used in the analyses as shown in Figure 4. The "real" time scale is used for implicit transient analyses: $t \in$ $\left[0, t_{\text {life }}\right]$. As explained before, a different time scale is used for the final explicit analysis: $\tilde{t} \in$ $\left[0, t_{\text {exp }}\right]$. In order to handle this difference, the time scale factor $\alpha_{s c}=\frac{t_{\text {life }}}{t_{\text {exp }}}$ is introduced and a change in the stress field variable is done by introducing $\tilde{\sigma}(\tilde{t})=\sigma(t)=\sigma\left(\alpha_{s c} \tilde{t}\right)$. Going back to the differential equations describing the multi-physical problem (Eqs. 1 to 6), it is possible to define a new system of equations with regards to the new scaled stress field variable $\tilde{\sigma}$. Hence, it is easy to demonstrate that new "scaled" parameters have to be defined whenever kinetic of the time is involved: $\widetilde{\tau_{l}}=$ $\frac{\tau_{l}}{\alpha_{s c}}, \widetilde{\tau_{c}}=\frac{\tau_{c}}{\alpha_{s c}}$ and $\widetilde{\eta_{v e}}=\frac{\eta_{v e}}{\alpha_{s c}}$ respectively for AAR and creep strains.

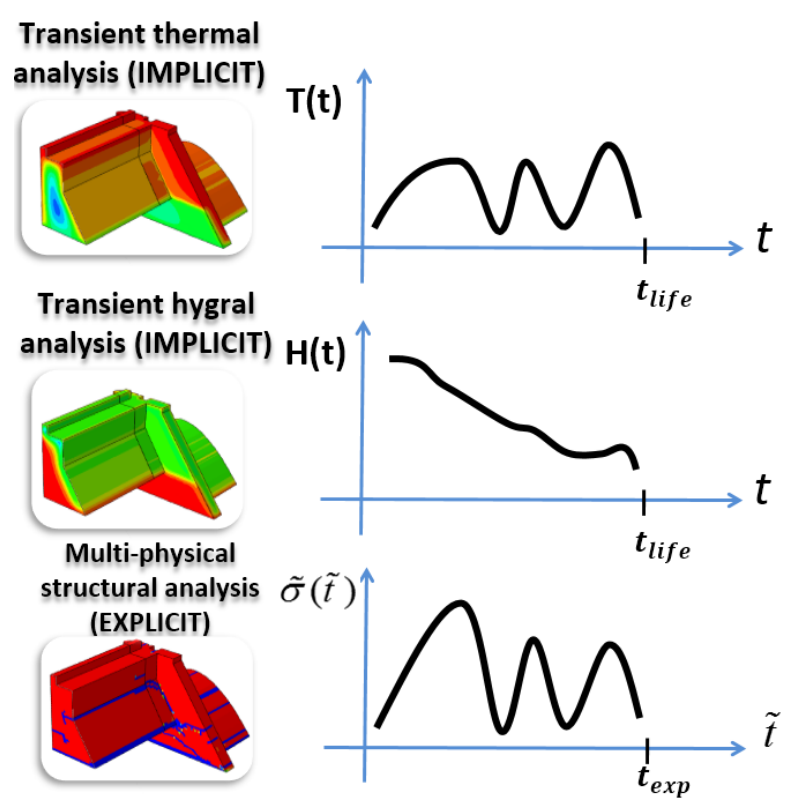

Figure 4: Difference in time scales

\subsection{Incremental formulation}

Due to the small increment size, the equation 6 can be rewritten as: $\dot{\xi}\left(t, T, \sigma_{0}\right)=f\left(t, T, \sigma_{0}\right)$.

A first possible and simple incremental formulation is to compute the rate of advancement of AAR $\dot{\xi}$ at each increment, knowing the value of $\mathrm{T}$ and stress state $\sigma_{0}$ and then use the integral to compute the total amount of AAR advancement:

$$
\xi(t)=\int_{0}^{t^{\prime}} f\left(t^{\prime}, T, \sigma_{0}\right) d t^{\prime}
$$


This will be called the first incremental formulation. As it will be seen later, this formulation ignores the cyclic variation of $\mathrm{T}$ and the high dependency of AAR advancement on it. Figure 5 demonstrates clearly this dependency for three different temperature values and more particularly the effect of temperature change which causes a shift of the rate of expansion to the right or left side. This temperature change can clearly result in an integral value of the rate (Eq. 7) more or less than the value of 1.0 that would be obtained in an isothermal case.

(a)

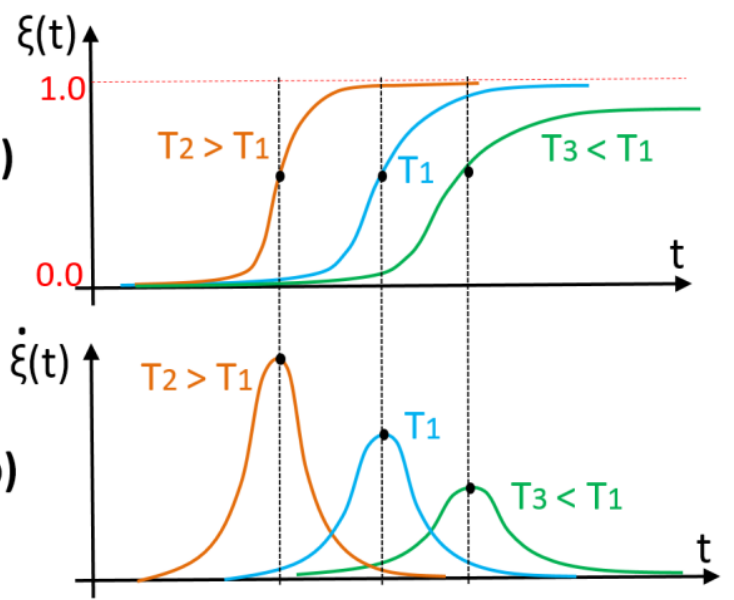

Figure 5: Effect of temperature variation

A new incremental numerical formulation is presented to correctly handle the dependency of the AAR kinetics on the temperature field in case of cyclic temperature variation. It will be called in this paper the second formulation. The idea is to compute at each new step $t_{0}+\Delta t$ the increment $d \xi$ of AAR advancement by using the isothermal curve $\xi$ at the previous increment $t_{0}$. A fictitious time $t_{0}^{*}$ is computed by equating the total AAR advancement between the two isothermal curves at steps $t_{0}$ and $t_{0}+\Delta t$ : $\xi\left(t_{0}^{*}, T\left(t_{0}\right)\right)=\xi\left(t_{0}, T\left(t_{0}+\Delta t\right)\right)$. This method is shwon schematically in Figure 6 for the case of a sudden temperature drop between the steps $t_{0}$ and $t_{0}+\Delta t$.

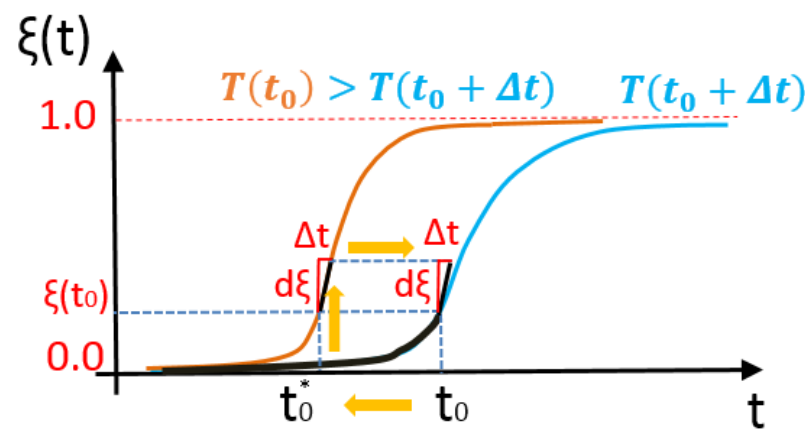

Figure 6: Effect of temperature variation

\section{VERIFICATIONS AT MATERIAL LEVEL}

A verification example is considered at the material point level of a single cubic element as shown in Figure 7.a. The example is taken from [19] page 53 with the same input data. All the corresponding results are shown in continuous lines in Figures 7.b to 7.c. A cyclic temperature field $T(t)$ is used as input to the multi-physical model. The time span used in the original reference model was 10 years whereas in the explicit model a $0.2 \mathrm{~s}$ analysis time is used, the time scale factor is therefore $\alpha_{s c}=1.5810^{9}$. The variations of the rate of the AAR $\dot{\xi}(t)$ and the total advancement of the AAR $\xi(t)$, outputs of the multi-physical model, are shown in Figures 7.c and 7.d. The results of the first formulation are represented in black whereas those of the second formulation are red-colored. The results using the first formulation match closely those reported in [19]. The use of the second formulation did not affect greatly the results for this case due to the small amplitude of temperature variation. The conclusions are different when the amplitude is increased from $1^{\circ} \mathrm{C}$ to $20^{\circ} \mathrm{C}$ which could be the case for example, for hydraulic structures in northern regions of Canada. This case is referred as modified example in Figure 7 and all the corresponding results are in dashed lines. A clear difference is seen in this case between the two formulations (Figures 7c and 7d). It can be demonstrated that the first formulation gives 
erroneous results by considering simpler examples of ramped temperatures. The problem occurs more precisely when the temperature change is "rapid" comparatively to the change in the value of the latency time affected by temperature according to Larive laws [2]. The change in latency time $\tau_{l}$ with respect to high or low values of temperatures (represented schematically by horizontal shift in Figure 5b) is also questionable.

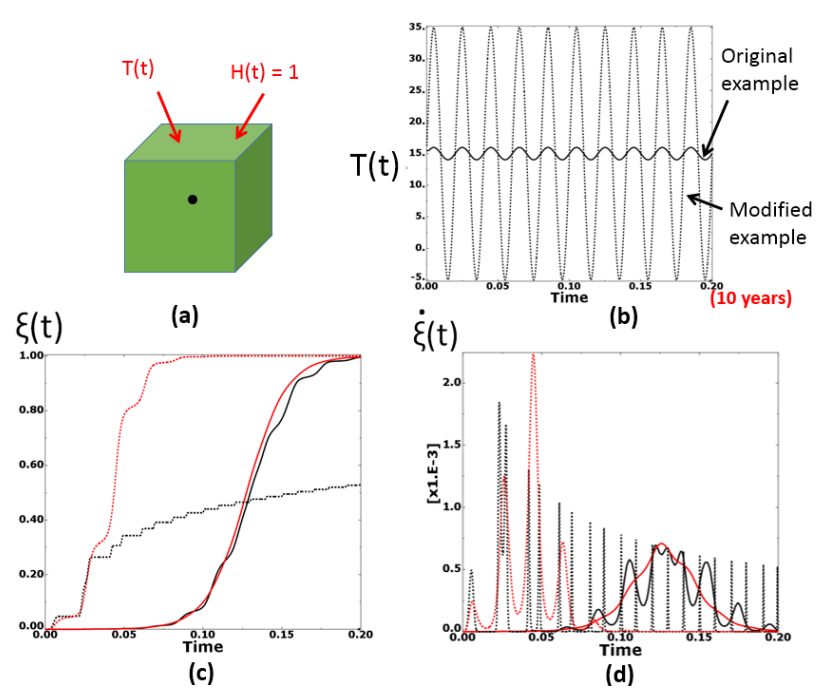

Figure 7: Verification example

\section{APPLICATION AT STRUCTURAL LEVEL}

The finite element model is shown in Figure 1 along with some dimensions. It is a portion of an existing hydroelectric facility that suffers from AAR. Only portions of the east gravity dam and the spillway are considered. The common structural problem in this case is the abrupt change in the lateral stiffness due to the presence of the hydraulic passage of the spillway. The pier of the spillway is subjected to the thrust action of the dam due to swelling reaction and behaves as a cantilever beam. Fixity condition is assumed at the bottom faces of the model and plain strain conditions are imposed at the lateral vertical faces of the dam and the spillway steed. Hydraulic loads are applied at the upstream faces with the normal closed condition of the spillway gates. The upstream water level is located 1.2 meters below the crest of the dam. The total period of analysis is 100 years. For the explicit model, the period of analysis was set to $10 \mathrm{~s}$. The fundamental mode of the model is around 0.05 $\mathrm{s}$ (upstream/downstream bending). It was decided to apply the mechanical loads (gravity and hydrostatic loads) quasi-statically within the first $1 \mathrm{~s}$ of the analysis, thus assuming a quasi-linear behaviour of the model in this first interval. The time scale factor is therefore $\alpha_{s c}=3.1510^{8}$. As a first approximation, only the AAR strains are considered additionally to the mechanical strains in Eq. 1. All other strains were neglected for this preliminary study. It is important to note here that preliminary data was used and that refinement of the model will be performed in future works. The objective here is to validate the feasibility of the methodology. For the mechanical constitutive law, the main input parameter used was the compressive strength of concrete of the real structure, all other parameters were derived accordingly. Table 1 gives some of the input data required by the STRAINCH and EPM3D subroutines. Latency and characteristic times were chosen in order to achieve $100 \%$ of AAR advancement within the analysis period of 100 years, under the computed temperature and relative humidity conditions (see Figure 8).

Table 1: Preliminary input data used for the multi-physical analysis

\begin{tabular}{lccc}
\hline \multicolumn{1}{c}{ Property } & Symbol & Value & Unit \\
\hline Mass density & $\rho$ & 2350 & $\mathrm{~kg} / \mathrm{m} 3$ \\
\hline Young modulus & $E$ & 25000 & $\mathrm{MPa}$ \\
\hline $\begin{array}{l}\text { Compressive } \\
\text { strength }\end{array}$ & $f_{c}^{\prime}$ & 25.0 & $\mathrm{MPa}$ \\
\hline Tensile strength & $f_{t}^{\prime}$ & 2.3 & $\mathrm{MPa}$ \\
\hline Poisson's ratio & $v$ & 0.18 & - \\
\hline $\begin{array}{l}\text { Mode I fracture } \\
\text { energy }\end{array}$ & $G_{F}$ & 0.2 & $\mathrm{kN} / \mathrm{m}$ \\
\hline $\begin{array}{l}\text { Long term } \\
\text { volumetric AAR } \\
\text { strain }\end{array}$ & $\varepsilon_{v \infty}$ & 0.002 & - \\
\hline
\end{tabular}




\begin{tabular}{lccc}
\hline $\begin{array}{l}\text { Reference } \\
\text { temperature }\end{array}$ & $T_{0}$ & 20 & ${ }^{\circ} \mathrm{C}$ \\
\hline Latency time & $\tau_{l}$ & 1150 & days \\
\hline $\begin{array}{l}\text { Characteristic } \\
\text { time }\end{array}$ & $\tau_{c}$ & 350 & days \\
\hline $\begin{array}{l}\text { Activation energy } \\
\text { for characteristic } \\
\text { time }\end{array}$ & $U_{c}$ & 5400 & ${ }^{\circ} \mathrm{K}$ \\
\hline $\begin{array}{l}\text { Activation energy } \\
\text { for latency time }\end{array}$ & $U_{L}$ & 9400 & ${ }^{\circ} \mathrm{K}$ \\
\hline $\begin{array}{l}\text { Compressive } \\
\text { limiting stress for } \\
\text { AAR }\end{array}$ & $\sigma_{u}$ & 10.0 & $\mathrm{MPa}$ \\
& & & \\
\hline
\end{tabular}

Input data required for the implicit transient analyses correspond to normal concrete in northern Canada environmental conditions. They are not exposed herein due to space limitation. Contrarily to most of previous works on hydraulic structures, a variable relative humidity $\mathrm{H}(\mathrm{t})$ is considered in this work. Sensitivity studies showed an important impact of this parameter on the damage pattern of the spillway pier. Figure 8 shows the evolution of the relative humidity and temperature fields with respect to virtual time for a given node on the dam. The advancement of the AAR, output of the multi-physical model is also shown in Figure 8.b.

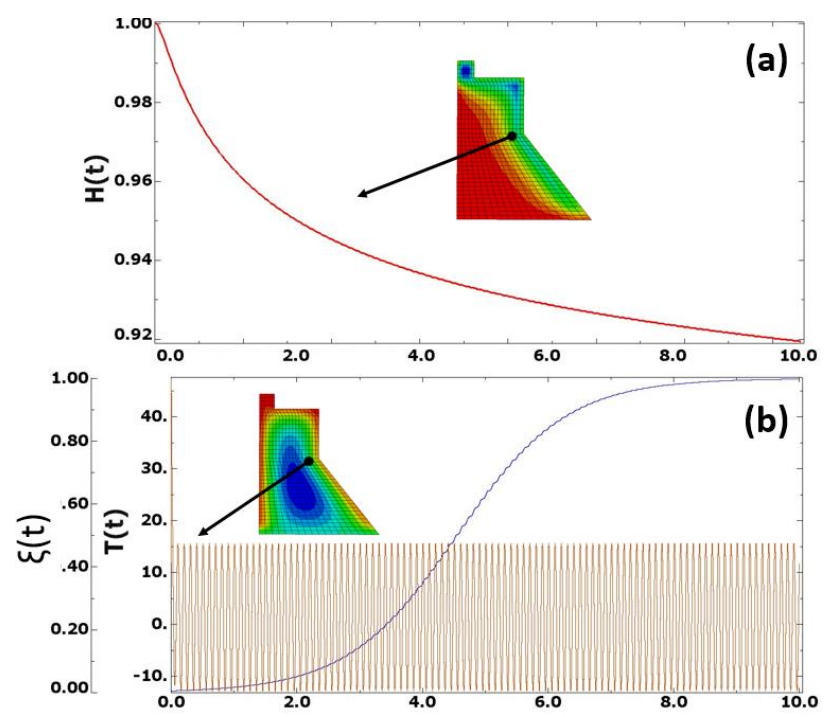

Figure 8: Evolution of: (a) relative humidity field; (b) temperature field and advancement of AAR
Some results are given in the following figures. The computation time for the multi-physical model was around eight hours on a standard quad-core laptop. Figure 9 shows the damage pattern evolution during the total analysis period of 100 years. The cracked elements are blue-colored whereas non-cracked elements are red-colored. A deformation scale factor of 50 is used for the deformed shape, which clearly shows the lateral bending of the pier due to thrust effect from the dam. Figure 10 shows a comparison between the real cracking pattern record (on a 3D virtual geometry) and the damage pattern of the multi-physical model. The similarity between the two is interesting though very preliminary data was used. This similarity was also observed for other hydraulic structural applications under study. As stated before, Figures 9 and 10 show a generalized damage pattern due to AAR, which is believed to cause convergence problems for conventional implicit solvers.

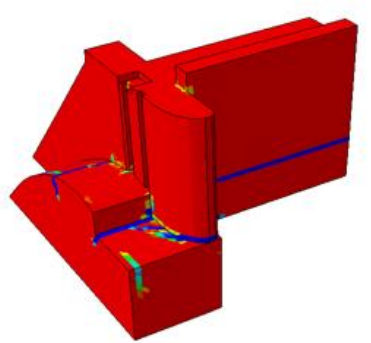

15 years

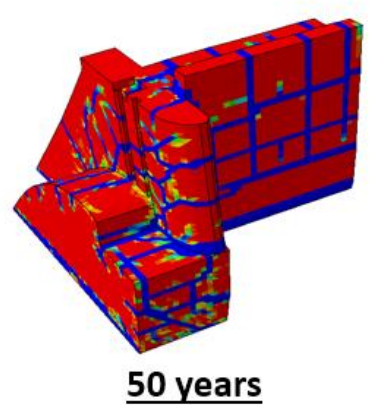

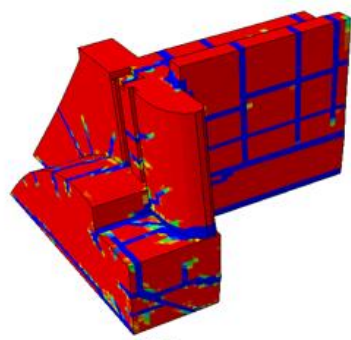

35 years

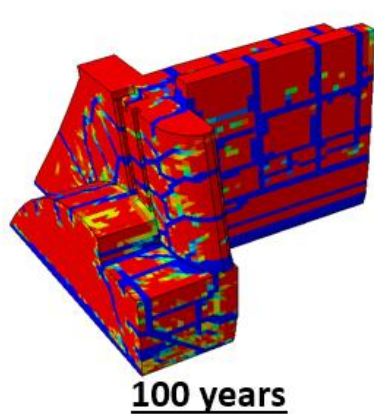

Figure 9: Damage pattern of the multiphysical model 


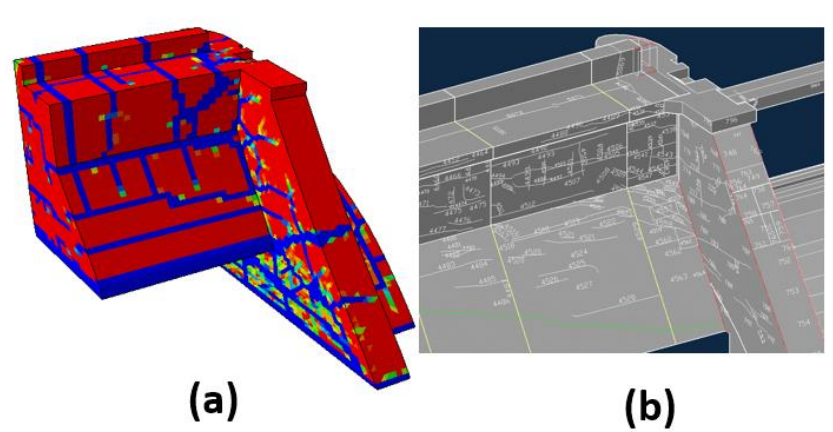

Figure 10: Comparison between: (a) damage pattern in the multi-physical model and (b) real cracking pattern record

\section{CONCLUSIONS}

A new computational framework is developed in this work for the multi-physical simulation of AAR in concrete structures using the explicit approach. A new scaling technique is developed in order to solve the problem of small time scale of the approach. The effectiveness and feasibility of the overall methodology is demonstrated via a real application example of a hydraulic structure.

\section{REFERENCES}

[1] Saouma, V., and Perotti, L. 2006. Constitutive model for alkali aggregate reactions. ACI Materials Journal, 103(3):194-202.

[2] Larive, C. 1998. Apports combinés de l'expérimentation et de la modélisation à la compréhension de l'Alcali-Réaction et de ses effets mécaniques. Thesis, LCPC, Paris.

[3] B. Massicotte, B., Nour, A., Ben Ftima, M., Yildiz, E., and Conciatori, D. 2012. EPM3D v 2.0, A user-supplied constitutive model for the nonlinear finite element analysis of concrete structures. Research report, École Polytechnique de Montréal.

[4] Léger, P., Côte, P., and Tinawi, R. (1996). Finite element analysis of concrete swelling due to alkali-aggregate reactions in dams. Computers\&Structures, 60(4),
601-611.

[5] Ulm, F., Coussy, O., Kefei, L. and Larive, C. 2000. Thermo-chemo-meachanics of asr expansion in concrete structures. ASCE Journal of Engineering Mechanics, 126(3): 233-242.

[6] Grimal, E., Sellier, A., LePape, Y. and Bourdarot, E. 2008. Creep, shrinkage, and anisotropic damage in alkali-aggregate reaction swelling mechanism part I: A constitutive model. ACI Materials Journal, 105(3).

[7] Comi, C., Fedele, R. and Perego, U. 2009. A chemo-thermo-damage model for the analysis of concrete dams affected by alkali-silica reaction. Mechanics of Materials, 41(3): 210-230.

[8] Sellier, A., Bourdarot, E., Multon, S., Cyr, M. and Grimal, E. 2009. Combination of structural monitoring and laboratory tests for assessment of alkali aggregate reaction swelling: application to gate structure dam. ACI Material Journal; pp 281-290.

[9] Multon, S. 2004. Évaluation expérimentale et théorique des effets mécaniques de l'alcali-réaction sur des structures modèles. Thesis, Université de Marne la Vallée, France.

[10] Ben Ftima, M. 2014. Utilisation de la méthode des éléments finis non-linéaires pour la conception des structures en béton armé : application aux structures massives, $\mathrm{PhD}$ thesis, École Polytechnique de Montréal, Canada.

[11] Prior, A. 1994. Applications of implicit and explicit finite element techniques to metal forming. Journal of Materials Processing Technology, 45(4): 649-656.

[12] Hibbitt, H.D., Karlson, B.I. and Sorensen, E.P. 2016. ABAQUS version 2016, finite element program. Hibbitt, Karlson and Sorensen, Providence, R.I., USA.

[13] Ben Ftima, M. and Massicotte, B. 2004. Introduction du modèle de Bouzaiene et Massicotte (1995) dans ABAQUS (Version 6.4.1 Standard et Explicit). Internal Report, École Polytechnique de Montréal, Canada.

[14] Ben Ftima, M. and Massicotte, B. 2015 a. Utilization of nonlinear finite elements for the design and assessment of large concrete 
structures, part I: Calibration and Validation. ASCE Journal of Structural Engineering, 141, 2015.

[15] Ben Ftima, M. and Massicotte, B. 2015 b. Utilization of nonlinear finite elements for the design and assessment of large concrete structures, part II: Applications. ASCE Journal of Structural Engineering, 141, 2015.

[16] Collins, M.P., Bentz, E.C., Quach, P. and Proestos, G. 2015. The challenge of predicting the shear strength of very thick slabs. Concrete International, November, 29-37.

[17] Martinola, G., Sadouki, H. and Wittmann, F. 2001. Numerical model for minimizing risk of damage in repair system. ASCE Journal of materials in civil engineering, November, 29-37.

[18] Pan, J., Feng, Y., Jin, F. and Zhang, C. 2013. Numerical prediction of swelling in concrete arch dams affected by alkaliaggregate reaction. European Journal of environmental and Civil Engineering, 17(4): 231-247.

[19] Saouma, V.E. 2014. Numerical Modeling of AAR. CRC Press Taylor \& Francis Group, London, UK. (300 pp.) 